\title{
The Immunitary Role in Cronic Prostatitis and Growth Factors as Promoter of BHP
}

\author{
luisetto $\mathbf{M}^{1 *}$, Behzad Nili-Ahmadabadi ${ }^{2}$ and Ghulam Rasool Mashori ${ }^{3}$, Ram Kumar Sahu ${ }^{4}$, Farhan $\mathrm{Ahmad}^{2}$ \\ Khan $^{5}$,Cabianca luca ${ }^{6}$ and Heba Nasser ${ }^{7}$ \\ ${ }^{1}$ Department of Pharmacology, Independent Researcher, Italy \\ ${ }^{2}$ Pharm D/PhD innovative Pharmaceutical Product Development Sspecialist, USA \\ ${ }^{3}$ Department of Medical \& Health Sciences for Woman, Peoples University of Medical and Health Sciences for Women, Pakistan \\ ${ }^{4}$ Columbia Institute of Pharmacy, India
}

${ }^{5}$ Professor \& Head, Department of Pharmacology, ESIC Medical College and Hospital (Ministry of Labour and Employment, Govt. of India), India

${ }^{6}$ Biomedical Laboratory, Italy

${ }^{7}$ Department of Microbiology \& Immunology, Heliopolis University, Egypt

Submission: April 10, 2018; Published: May 03, 2018

*Corresponding author: Iuisetto M, Department of Pharmacology, Independent Researcher, Email:Italy maurolu65@gmail.com

\begin{abstract}
Aim of this work is to verify relationship between immune systems and chronic prostatitis and BHP progression. A vicious cycle that involve chronic flogosis, tissue remodeling, grow factors, inhibition of apoptosis, and other phenomena. A deel knowledge in this pathogenesys make possible to serch new pharmacological strategiers, to improve actual therapy.
\end{abstract}

Keywords: BPH; Medicinal chemistry; Clinical pharmacy; Drug design; Drug delivery

\section{Introduction}

From biomedical litrerature is reported that related BHP progression some factors are deeply involved: Bacterial/ virus prostate infection, chronic flogosis,hormonal factors, auto immune reactions, urinary reflux, kind of diet, sedentary behavior pelvic congestion, proctities, obstruction prostate duts, microcalcification (Bcateria can come across also lymphatic way (from rectum), by ematic way, rising uretral infectious). All these events contribute to give an increased production of flogosis mediators, growth factors (stromal and epithelial) in a vicious cycle increase the pathologic condition.

The immune status of the prostate gland include for example linphocite $\mathrm{T}$ and macrophages infiltration related to flogosis cytokine hyper production.

This reactions with cellular damage produce ROS reactive oxigenes species, increase in production of growth factors (VEGF, IL 8 FGF 7 , TGFB, FGF 2 and other). Inhibition in apoptosis and tissue remodeling with hyper production of extracellular matrix and stromal parts.

Other factor can be responsible in relapse in chronic prostatitic in BHP in example micro calcifications and dust obstructions. Other condition that can be involved can be metabolic syndrome of diabetes and related biochemical and hormonal modify in metabolisms. The same also all the pathology involved by nervous systems and bladder, urethral restrictions and other urology disease.

Biofilm presence that makes more antimiciorial resistances reduced urinary prostatic fluid in example by IPB, urinary reflux (chemical cystitis and prostatitis), prostatic cancer and other factors.

Adrenergic hypertonus of prostate capsule contribute to phisio-pathology. Involved often Intestinal functional abnormalities and pathologies, ano-rectal, sexual transmission infectious and also due to bacterial sanctuaries and intra prostatic calcifications. We have see that a bacterial disease can be primitive or secondary (bacteria cause of infection or sovrainfectious in a flogosis tissue or to other conditions) in example hypertonus in pelvic sfinteric muscle.

In The actual medical therapy of BPH we can see: antibiotics, alfa blokers, 5-ARI, fitothearapic (Serenoa repens) with different clinical functions and activities and other molecule as fans (local or systemic), cortisones.

The aim of this review work is to observe some relevant literature in our opinion related the management of BHP and progression under a pharmaceutical - endocrinologic - metabolic disease and immunologic point of view. 
A deep knowledge in the pharmaceutical properties of some molecule (antimiciobials, antiflogosis, Antiedemigen, alfa blochers, 5- ARI and other instruments) can help the physicians in the right choice.

\section{Material and Methods}

Using a review method and observing some literature in biomedical database can be produced a new approach in pharmacological strategies in some relevant urologic disease or to prevent it.

Observing some works related to pathology like BPHP we to try find NEW strategies that could be translated in order to achieve better clinical results adding new drug delivery system or other improvement in molecular structure in order to achieve more efficacy and reduced relapses or surgical needs.

\section{Results}

\section{From literature we can find}

"In this issue of BJU International, Gandaglia et al. summarize the evidence supporting the role of chronic prostatic inflammation in the pathogenesis and progression of BPH. Briefly, one or more concomitant factors (bacterial infections, viruses, sexually transmitted organisms, dietary factors, hormones, autoimmune response and urine reflux) can stimulate an inflammatory reaction in prostatic tissue characterised by infiltration of T-lymphocytes, activation and up-regulation of pro-inflammatory cytokines, increased expression of potent stromal and epithelial growth factors (e.g. fibroblast growth factor, FGF-2) and consequently abnormal proliferation of prostatic cells. Moreover, local hypoxia plays an important role stimulating reactive oxygen species (ROS) release, Continuous repair gives tissue remodeling.

Neo-vascularization processes and the production of other additional growth factors (vascular endothelial growth factor, interleukin 8, FGF-7, TGF- b and FGF-2). Interestingly, this mechanism is self-perpetuating, creating a local vicious cycle.

Available clinical data seems to emphasise the prevalence of chronic prostatic inflammation in BPH. Indeed, a sub-analysis of the REDUCE (Reduction by Dutasteride of prostate Cancer Events) trial shows that in patients with $\mathrm{BPH}$ a chronic prostatic inflammation can be detected in $77 \%$ of patients who underwent prostate biopsies.

Moreover, this study also showed a statistically and clinically significant correlation between chronic prostatic inflammation and LUTS severity, especially when the storage subscale was considered. As extensively described by Gandaglia et al., other studies have shown a significant correlation between chronic prostatic inflammation and prostate volume and an increased risk of acute urinary retention. Obviously, chronic prostatic inflammation can be histologically detected only in patients who undergo prostate biopsies for suspicion of prostate cancer. However, most patients with LUTS/BPH do not undergo a prostate biopsy. For this last category, the use of specific biomarkers correlated with chronic prostatic inflammation has been proposed as a potential alternative. Although interleukin 8 seems to be the most reliable and predictive surrogate marker to identify patients with chronic prostatic inflammation, its use is not yet popular, it is expansive and probably requires further clinical evaluation before introduction into daily clinical practice. In this context, the detection of prostatic calcifications can represent a simple ultrasound sign to suspect the presence of chronic prostatic inflammation.

In patients aged $>50$ years, prostatic calcifications represent an age-related alteration of the prostatic fluid. Prostatic calcifications can produce an obstruction of the intraprostatic ducts stimulating an inflammatory response in prostatic tissue characterized by lymphocyte infiltration, cytokine activation and ROS release. This results in damage of epithelial and stromal prostatic cells and a subsequent process of wound healing consisting of stromal proliferation and excessive extracellular matrix production; summarizes these mechanisms following prostatic duct obstruction. I think that in patients with prostatic calcifications and severe LUTS (with predominant storage symptoms) the presence of chronic prostatic inflammation should be strongly considered.

Patients with high-grade prostatic inflammation seem to have a worse response to traditional medical therapy for LUTS/BPH (a -adrenergic blockers and 5a -reductase inhibitors) compared with patients without or with low-grade prostatic inflammation [5]. Indeed, neither a-adrenergic blockers nor $5 \mathrm{~A}$-reductase inhibitors have an anti-inflammatory effect. Therefore, drugs commonly used for the treatment of non-neurogenic LUTS cannot influence the described chronic inflammatory status.

An anti-inflammatory effect on human prostate has been ascribed to the hexanic lipidosterolic extract of

Serenoa repens. Specifically, in 2003 Vela Navarrete et al. [2] reported a significant reduction of interleukin 1 and TNF- a levels 3 months after Serenoa repens treatment compared with the placebo arm. Recently, Latil et al. [3] showed thatSerenoa repensinhibits the expression of two" [3]

- $\quad$ "Dutasteride showed greater efficacy in reduction of TPV and PSA and in symptomatic improvement by IPSS score than finasteride."[15] (Remember that flogosis can results in partial inefficacy of 5-AR1)

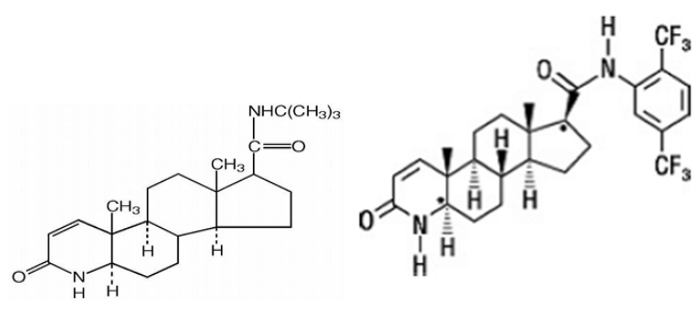

FINASTERIDE DUTASTERIDE 
See the different chemistry formula and related pharmacological profile of action (reversible or irreversible activity in 5-HT INIBITION) but also related to other factors as tissue penetration related chemical groups ad lipophilic balances

- $\quad$ "It is clearly known that the prostatic gland is frequently involved in Different pathologies in adults and elderly patients. Benignant or malignant: anatomic of functionally disease. Frequently other condition as bladder dysfunctions can be associated or added to this pathologies or caused by it. Some of these pathologies give low level in patient quality life and reducing in life expectance (malignant). Malignant pathologies start as local disease but can diffused as metastatic interesting other apparatus of the patient (patient frequently show resistance to first line therapy in example hormonal blocks, or different chemotherapies). But, In all of this pathology we can see a not complete resolution with current medical therapy in all patients and this can be related with the difficulties of some drugs to penetrate adequately in the tissue. In example we can see that I many benignant cases as HYPERTROPHIA also phytotherapic produces or dietetic integrator are frequently added by the specialist to the classic drugs polytherapy. This shows that the classic drug therapy can be improved. In example Relapses in bacterial chronic prostatitis are commonly in great number of patient even under the best pharmacological therapies available today. It means that something goes wrong in this kind of therapy [4]."

\section{- $\quad$ "According Biomedical Literature}

Responsible of relapses of chronic prostatitic and related relapses can be: biofilm, reduced urinary prostatic fluss in example by IPB, urinary reflux (chemical cystitis and prostatitis), prostatic cancer and other factors.

a. Biofilm is responsible about difficulties in diagnostic methods and in therapy.

b. Bacterical prostatities is associated in frequent E.Coli infectious, enterococcus faecalis but other germs

c. Can be involved (example Clamidia, Micoplasm).

d. Involved often intestinal functional abnormalities and pathologies, ano-rectal, sexual transmission infectiousand also due to bacterical sanctuaries and intraprostatic calcifications.

e. We have see that a bacterial disease can be primitive or secondary (bacteria cause of infection or sovrainfectious in a flogosis tissue or to other conditions) in example hypertonus in pelvic sfinteric muscle.

f. We have see the that infectious can be across different process: Trought ematogenic, lymphatic, contiguity, local pathology, sexual way, trans uretral UTI, GI pathologies, proctities, neoplasia, prostatic reflux, rectal bacteria linfatic diffusion and other. g. Medical examination and Ecografy make possible to evaluate local situation in order to exclude other pathologies.

h. Ascessual evolution, micro litiasis and other tissue abnormalities.

i. Acute disease can develop in chornic evolution, with ascessual possibilities (needed surgical drainage) Only the Antimicrobial therapy efficacy only in little prostatic ascessual Cavity.

\section{Prostatic ascess contribute to obstruction" [7]}

"The role of infiltrating cells (I.C.), commonly observed in the adenoma interstitial tissue, is unknown. We tested the hypothesis that I.C. are related with BPH progression by: phenotypically characterizing these cells; quantifying the expression of lymphokines and growth factors; investigating the response to Permixon (P) in a clinical study. Permixon is a lipido sterolic extract of Serenoa repens possessing pharmacological activities and widely used in the treatment of men with BPH.

HISTOLOGICAL: A difference was observed in the number of lymphocytes B between C $(91.4 \pm 44.1)$ and P treated (58.2 \pm 53.7$)$ groups $(\mathrm{p}=0.097)$. BIOLOGICAL MARKERS: TNFalpha and IL1beta were dramatically lower in the Permixon treated group. Other parameters did not show significant changes. CLINICAL: IPSS in the Permixon treated group was significantly reduced $(p<0.006)$ from $20.0+5.9$ to $14.9+3.8$ after three months of treatment.

The BPH inflammatory hypothesis was tested in humans. Our pilot study shows a significant reduction of some inflammatory parameters in prostatic tissues of patients treated with Permixon. These biological findings justify a pharmacological effect of this drug on the inflammatory status of the adenoma. A correlation with clinical improvement was observed [2]."

"What's known on the subject? and What does the study add? Pervasive inflammatory infiltrates, mainly composed of chronically activated $\mathrm{T}$ cells and monocytes/macrophages, have been observed in benign prostatic hyperplasia (BPH). Permixon $\AA$, a hexanic lipidosterolic extract of Serenoa repens (hexanic LSESr) used to treat urinary dysfunction in BPH patients, has anti-inflammatory activities. This paper provides new insights into the anti-inflammatory properties of Permixon ${ }^{\circledR}$. We report that hexanic LSESr inhibits early steps of leukocyte infiltration in vitro by down regulating MCP-1/CCL2 and VCAM-1 expression.

To investigate the mechanisms by which hexanic lipidosterolic extract of Serenoa repens (hexanic LSESr) may prevent leukocyte infiltration in benign prostatic hyperplasia by studying its impact on monocyte chemo attractant protein $1 /$ chemokine (C-C motif) ligand 2 (MCP-1/CCL2) and vascular cell adhesion molecule 1 (VCAM-1) expression in vitro.

After pretreatment with hexanic LSESr, human prostate (epithelial and myofibroblastic) cells and vascular endothelial 
cells were stimulated with proinflammatory cytokines. MCP-1/ CCL2 and VCAM-1 mRNA expression was quantified by real-time PCR. ELISA kits were used to determine MCP-1/CCL2 levels in culture supernatants and VCAM-1 expression in living cells.

Hexanic LSESr reduced MCP-1/CCL2 mRNA levels in both epithelial (BPH-1) and myofibroblastic (WPMY-1) prostate cell lines. Hexanic LSESr down regulated MCP1/CCL2 secretion by WPMY-1 cells in a concentration-dependent manner, more efficiently than Serenoa repens extracts obtained by supercritical carbon dioxide extraction. Hexanic LSESr inhibited tumornecrosis-factor- $\alpha$-induced MCP-1/CCL2 secretion by the human vascular endothelial cell line EAhy.926, as well as surface VCAM1 protein expression, in a concentration-dependent manner.

Hexanic LSESr impedes key steps of monocyte and T cell attraction and adherence by inhibiting MCP-1/CCL2 and VCAM-1 expression by human prostate and vascular cells in an inflammatory environment. These findings provide new insights into the anti-inflammatory effects of the hexanic lipidosterolic extract of Serenoa repens, Permixon $\AA$, in benign prostatic hyperplasia [3].

"Randomized controlled trials suggest an increased risk of heart failure with dutasteride, which inhibits both the type 1 and type 2 isoforms of $5 \alpha$-reductase. In contrast, no such association has been suggested for finasteride, which selectively inhibits the type 2 isoform. We investigated the risk of cardiovascular events among patients receiving dutasteride relative to finasteride.

We performed a population based cohort study of Ontario men 66 years old or older who commenced treatment with dutasteride or finasteride between October 1, 2005 and March 31, 2015. For each individual treated with dutasteride, we identified 1 treated with finasteride, matching on a propensity score and calendar quarter of treatment initiation to account for temporal changes in prescribing. The primary outcome was hospitalization for heart failure. Secondary analyses were done to examine acute myocardial infarction and stroke. Cox proportional hazards regression was used to adjust for differences between groups.

We studied 36,311 men who commenced dutasteride and 36,311 treated with finasteride. In the primary analysis, we found no difference in the risk of heart failure among patients receiving dutasteride relative to those receiving finasteride (adjusted HR 0.98, 95\% CI 0.88-1.08). Similarly, we found no difference in the risk of acute myocardial infarction (HR 0.94, 95\% CI 0.82-1.08) or stroke (HR 1.03, 95\% CI 0.88-1.20).

In this population based cohort study of more than 72,000 older men, dutasteride was not associated with an increased risk of cardiovascular events relative to finasteride [6]."

"A recently published large, long-term randomized controlled trial (RCT) brought into question the safety of dutasteride after a significantly increased risk of 'cardiac failure' was noted in the dutasteride arm of the trial compared with placebo. Our objective was to perform a meta-analysis to assess the risk of cardiovascular adverse events with the use of dutasteride for the prevention or treatment of prostatic disease.

We searched MEDLINE and EMBASE, unpublished articles identified through FDA/EMEA websites, study registers of pharmaceutical companies and reference lists of articles. Parallel-group, randomized controlled trials where men received dutasteride for the prevention of prostate cancer or treatment of prostatic hyperplasia against any comparator intervention were included. Heart failure was the primary outcome of interest but we also looked at myocardial infarction and stroke. Fixed-effect meta-analysis of pooled relative risk (RR) ratios of adverse effect outcomes was conducted.

In all, 12 RCTs were included in the meta-analysis after detailed screening of 564 citations. The total number of participants was 18,802, and study duration ranged from 6 to 208 weeks. Only two trials provided details on adequate allocation concealment, whereas all the trials stated they were double blind in nature. Dutasteride was not associated with a statistically significant increased risk of heart failure (RR 1•05; 95\% confidence interval $[\mathrm{CI}], 0 \bullet 71-1 \bullet 57, \mathrm{I}(2)=20 \%)$, myocardial infarction (RR 1•00; 95\% CI 0•77-1•30, I(2) = 0\%) and stroke (RR, $1 \bullet 20 ; 95 \% \mathrm{CI} 0 \bullet 88-1 \bullet 64, \mathrm{I}(2)=0 \%)$ as compared to controls.

We did not find consistent evidence of a significant association between dutasteride therapy and the risk of cardiovascular adverse events [16].

- $\quad$ "Dutasteride significantly improved Qmax, reduced IPSS score, and improved Quality of Life as compared to Finasteride at the end of the 12 -week period. Dutasteride with its inhibitory effects on type 1 and 25 alfa reductase, produces significantly better results than Finasterid [8].

- "On multivariable logistic regression analysis, dutasteride was the only factor that significantly reduced clinical progression of benign prostatic hyperplasia, with an odds ratio of 0.47 (95\% confidence interval 0.37 to 0.59 , $\mathrm{P}<0.001$ )" [9].

- $\quad$ "Chronic inflammatory diseases have been shown to be associated with NF-kappa B activation and impaired apoptosis of immune cells. The aim of the present study was to investigate

a. If sulfasalazine and its colonic metabolites 5-aminosalicylic acid (5ASA) and sulfapyridine affect NFkappa B/Rel activation and viability of T-lymphocytes.

b. Sulfasalazine inhibits NF-kappa B/Rel activation in the murine T-lymphocyte cell line RBL5 using electrophoretic mobility shift assays. In transfection assays sulfasalazine treatment for $4 \mathrm{~h}$ inhibits kappa B-dependent transcription with an IC50 value of approximately $0.625 \mathrm{mM}$. 
c. Higher doses or prolonged treatment result in cell death of T-lymphocytes in a dose- and time-dependent manner. Cell death is caused by apoptosis as judged by DNA fragmentation, annexin $\mathrm{V}$ and Apo 2.7 staining. Induction of apoptosis is a fast event with $50 \%$ apoptotic cells after $4 \mathrm{~h}$ incubation with $2.5 \mathrm{mM}$ sulfasalazine. The ED50 value for apoptosis induction after $24 \mathrm{~h}$ treatment was approximately $0.625 \mathrm{mM}$.

d. In contrast, 5ASA and sulfapyridine neither inhibit NFkappaB/Rel activation nor induce apoptosis in T-lymphocytes at doses up to $5.0 \mathrm{mM}$.

e. These results demonstrate that sulfasalazine, but not 5ASA or sulfapyridine, strongly inhibits NF-kappaB activation and potently induces apoptosis in T-lymphocytes. Inhibition of NF-kappaB/Rel activation and subsequent clearance of activated T-lymphocytes by apoptosis might thus explain the beneficial effects of sulfasalazine in the treatment of chronic inflammatory disorders [10].

Mesalazin (inibit nf-kb - induce apoptosis in linph. T)

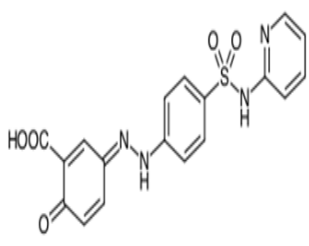

Sullasalazin

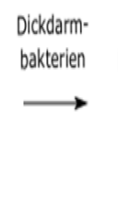

Mesalazin

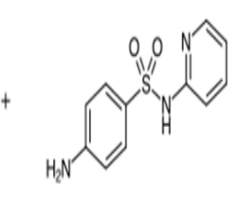

Sullapyridin
See also in example: United States US 20040198826A1 Patent Application Publication Pub. No.: US 2004/0198826A1 Baiker et al. [12] Pub. Date: Oct. 7, 2004 PHARMACEUTICAL COMBINATION FOR TREATING BENIGN PROSTATIC HYPERPLASA OR FOR TREATING ABACTERIAL PROSTATITIS: "0018 Within the meaning of the present invention, preferred non-Steroidal inflammatory drugs are the cyclo-oxy US 2004/ 0198826 A1genase (COX) inhibitors, such as the non-selective COX inhibitors acetylsalicyclic acid, mesalazin, ibuprofen, naproxen, flurbiprofen, fenoprofen, fenbufen, ketoprofen,et other" [11].

"Chronic bacterial prostatitis (CBP) is a persistent infection of the prostate characterized by poor quality of life mainly due to frequent relapse episodes caused by incomplete eradication of causative pathogens. Aggressive antibacterial therapy is required to attenuate the severe symptoms of CBP and to achieve a permanent cure. Although fluoroquinolones are currently recommended as first-choice agents, macrolide antibiotics are emerging as a noteworthy option for the treatment of CBP. Macrolide antibiotics are characterized by an impressive array of distinct pharmacokinetic (PK) and pharmacodynamic (PD) properties. These properties include high intracellular accumulation in phagocytes and at sites of infection, including the prostate; broad antibiotic but also biofilm-inhibiting properties; immunomodulating and inflammation-resolving activities. These features offer particular advantages for the treatment of chronic infections of the prostate gland, which are not easily amenable to drug therapy. Macrolides may be exploited to counteract the unsatisfactory rates of clinical symptom improvement and pathogen eradication. The results of a number of clinical trials support this proposal [12].

"We analyzed the incidence of anal disease in patients with nonbacterial prostatitis (NBP) or with prostatitis-like syndrome (PLS), and evaluated the clinical efficacy. The complicated rate of anal disease in these patients was $29.7 \%$ (31.8\% for NBP and $28.1 \%$ for PLS), and the overall incidence of active anal disease was $15.4 \%$ (16.2\% for NBP and $14.8 \%$ for PLS), it yielded a significantly higher complicated rate than other urological disease ( $p$ less than 0.01). The most common type of anal disease was hemorrhoids, especially piles. The clinical cure rate for anal disease in NBP patients was $71.4 \%$, and in PLS patients was $58.2 \%$. The high incidence of hemorrhoids (especially piles) was in these patients by clinico-statistical observation suggests that the development of anal disease may be etiologically correlated with NBP and PLS. Furthermore, we noted that Kampo treatment (Keisibukuryogan) was useful in the treatment of prostatitis complicated by anal disease, especially when combined with anti-hemorrhoidal suppositories against active anal disease in PLS patients (p less than 0.05) [13].

"The pathogenesis of nonbacterial prostatitis (NBP) is not understood mainly due to the lack of appropriate experimental models. We developed a new experimental model of NBP by inducing a partial obstruction of the urethra (PUO) in the rat. Male Wistar rats aged 12 weeks were used. PUO was produced by a nylon ligature on the urethra over a rubber tube. The tube was slipped out after the ligature had been tied. Two rats were examined histologically 6h, 1 day, 3 days and 7 days after PUO. In another group, two rats were killed at 1, 3 and 7 days after the release of the PUO that had been left in place for 3 days. On day 3 , another eight rats with PUO and eight control rats had $2 \mathrm{ml}$ of urine in the bladder replaced by the same volume of lucifer yellow (LY; 10microg/ml, MW 500), microperoxidase (MP; 20microg/ $\mathrm{ml}$, MW 1900), horseradish peroxidase (HRP; $10 \mathrm{microg} / \mathrm{ml}$, MW 40,000), or saline as control, respectively. Lymphocytic infiltration and interstitial edema were noted in the prostate following PUO, being most prominent on day 3. After the release of the PUO, these inflammatory changes gradually disappeared. Only LY was noted within the prostatic stroma of the rats $2 \mathrm{~h}$ after bladder instillation. Intraprostatic urinary reflux may be an etiologic factor in NBP. The present study showed that lower urinary tract obstruction caused NBP in the rat. Penetration of prostatic tissue by low-molecular-weight substances in the urine may trigger NBP [14]."

\section{Discussion}

In BHP we can observe a great damage to the patient quality of life and cost for public institutions and insurance. We must consider that BHP disease under an infective, endocrine and immune phenomena deeply interconnected. 
Non pharmacological starategies can be Kegel exercise to reduce pelvic hypertonus, lifestyle, dietary, phisyca activity adn other factor can reduce relapsis in chronic and acute prostatitis related in $\mathrm{BPH}$, reduction of pelvic congestion.

No sedentariety, no stinging spicies, no etilic alcool drinks, no cured meats et other. The the Intestinal flora situation is relevant to prevent exchange of bacteria form rectum, proctological disease, hemorrhoids.

In relapses of BHP as chronic or acute prostatitis factors like different kind of bacteria population in the infectious and related activity profile of antimicriobial used (gram + , gram -, micoplasm and calmidya, herpes virus ), profile of resistance, biofilm presence, tissue hyperplasia, chronic flogosis, autoimmunity , kinetics of tissue penetration etc. must be high considered to achieve the best results .

Also the receptor local situation in bladder and trigone (adrenergic, cholinergic) are relevant factors useful in therapy strategy to improve urinary flow. (as wee as anatomic obstruction of prostate uretra) and other.

Under the light o reported literature Prostate gland must be considered an immunologis subject in some disease at BHP and crhornic prostatitis and this aspect must not to be overlooked especially in therapy.

Examples of some antimicrobials used (only few examples)<smiles>O=C(O)C1CC(C2CC2)C(O)C2C=C(F)C(N3CCNCC3)C=C12</smiles>

CIPROFLOXACIN - fluorochinolons, other LEVOFLOXACIN

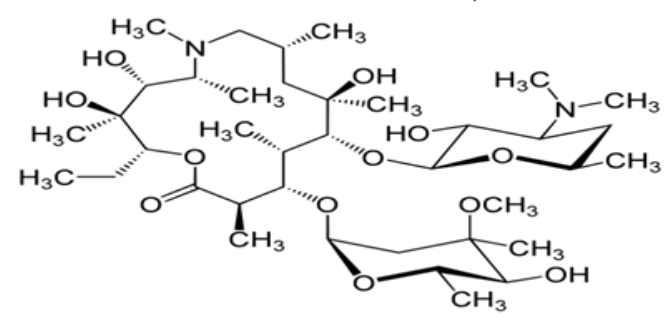

\section{AZITROMICIN, macrolides}

See property in biofilm action and profile of efficacy / effectiveness

In resistances profile Other antimicrobials as, ampicillin, carbenicillin, doxicillin, gentamicin, imipenem, phyperacillina tazob., Fosfomicin parenteral and other

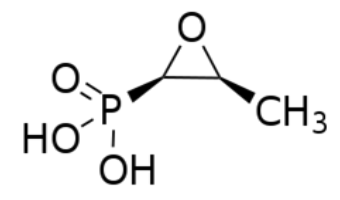

fosfomicin
SERENOA REPENS alcaloids

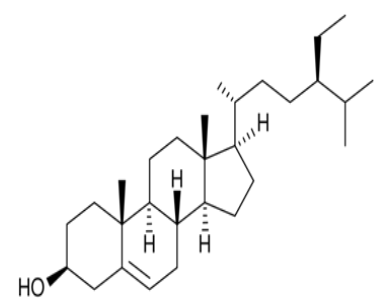

Beta fitosterols

In this paper we have see only some molecule currently in use to shaw the relationship between dynamics, kinetics, drug delivery strategy and medicinal chemistry properties.

Prostate gland is considered pharmacological sanctuaries and for this reason using the best strategies it can result in more $\%$ of global clinical efficacy.

Rigth spectrum anti microbial covering, right time of the cure, the right antiflogosis and anti edemigen therapy associated to 5- ARI to induce apoptosis and alfa blokers can be the golden endopoint to prevent the progression of BHP.

Also a good blood glucose control prevent recurrent urinary infectious (glucose is normally used by microbs growth) and uric acid blood monitoring and control and therapy can be useful to reduce global stinging Situation (crystals).

\section{Conclusion}

From literature we can see some relevant implication in pharmacological therapy related to medicinal chemistry of some drugs currently in use.

In medical approach the right profile of action (dynamics, antibacterial spectrum, dosage, duration of therapy, pharmacokinetics, tissue penetration and other characteristics are to take in great consideration in this kind of situation.

Drugs strategies that must consider antimicrobial power, antimicrobial concentration, alfa bloccants effect, 5 HT inhibitions, anti flogosis but also new drug delivery systems

Anti-edemigen, antioxidant et other as well as preventive non pharmacological strategies like right water intake, physical activity, no traumatic sports, not sexual abuse but regular (spermatozoa degradation products), and other.

From literature we have see the prominent role played by chronic flogosis, as well as hormonal unbalance that produce tissue remodeling, hyper production of growth factors and reduced apoptosis.

All factors involved in BHP progression.

A deep knowledge also in medicinal chemistry of molecule currently used make possible a more rational therapy of chronic prostatitis as well as BHP. What is relevant is that all chronic 
prostatitis must be treated in the right way since first time to prevent chronic progression and the vicious circle.

Can we think new delivery system to achieve better improvement in local tissue penetration of Therapeutics principles?

We know that it clear that the drugs vehicle is important as the same drugs and that modifying the molecule we can improve the activity in relevant way also due by different tissue diffusion (In example see the chinolons and fluorochinolons different pattern of tissue penetration) and in duration of action time. As request to prevent relapses in acute and chronic prostatitis.

Lipophilic hydrophilic balances, acido-bases properties, molecular weight and other pharmaceutical characteristics great influence the pk. Kinetics as VD or T1/2 but also the profile of linked chemical groups can give new delivery of classic drugs (Other can be acid or basic molecular properties or velocity in metabolism).

We know that for example in the same drug classes in examples chinolons little chemistry modify can results in improvement of efficacy so we can think that little chemical modify o new delivery system can produce better clinical results by a better pharmacokinetics in this sanctuaries.

We can consider innovative drug delivery systems to improve clinical efficacy reducing global toxicity.

Novel loco-regional drug delivery systems or more targeted to prostatic tissue, more time extended drug Presence in prostatic tissue, using of innovative technologies as colloidal micro- nanosystems and other, better biofilm disaggregation properties, modifying lipofilic - idrofilic molecular balances, acido-bases properties of molecule, add of more useful chemical groups to classic formula to improve kinetics and prostatic intake, implants, and other medicinal chemistry new technologies that can add advantages vs the actual therapy models.

All this strategies must be added to adeguate flogosys control and towards preventing lymphocyte $\mathrm{T}$ and macrophages prostate infiltration and an efficacy grow factors control.

\section{Clarifi cations}

This paper was not writed for any diagnostic or therapeutic intent, only to produce new research hypotesys.

\section{References}

1. Luisetto M, Behzad NA, Ghulam RM (2017) Relapses and Recurrent Chronic Bacteric Prostatitis - Biofilm Related, A Case Report. J of Pharmacol \& Clin Res 4(4): 555644.
2. Navarrete VR, Cardoso GJV, Barat A, Manzarbeitia F, Farré LA (2003) BPH and inflammation: pharmacological effects of Permixon on histological and molecular inflammatory markers. Results of a double blind pilot clinical assay. Eur Urol 44(5): 549-555.

3. Latil A, Libon C, Templier M, Junquero D, Lantoine-Adam F, et al. (2012) Hexanic lipidosterolic extract of Serenoa repens inhibits the expression of two key inflammatory mediators, MCP-1/CCL2 and VCAM-1, In vitro. BJU Int 110(6 Pt B): E301-307.

4. Luisetto M, Nili-Ahmadabadi B (2017) Chronic Prostatitis: The Clinical Pharmacist Role and New Delivery Systems. J Bioanal Biomed 9: e151.

5. Ficarra V (2013) Is chronic prostatic inflammation a new target in the medical therapy of lower urinary tract symptoms (LUTS) due to benign prostate hyperplasia (BPH)? BJU International.

6. Skeldon SC, Macdonald EM, Law MR, Huang A, Paterson JM, et al. (2017) The Cardiovascular Safety of Dutasteride. J Urol 197(5): 13091314.

7. YK, Ho R, Smith M, Wong O, Sandhu M, et al. (2013) Systematic review evaluating cardiovascular events of the 5-alpha reductase inhibitor Dutasteride. J Clin Pharm Ther 38(5): 405-415.

8. Ravish IR, Nerli RB, Amarkhed SS (2007) Finasteride to Evaluate the Efficacy of Dutasteride in the Management of Patients with Lower Urinary Tract Symptoms and Enlarged Prostate. Arch Androl 53(1): 17-20.

9. Toren P, Margel D, Kulkarni G, Finelli A, Zlotta A, et al. (2013) Effect of dutasteride on clinical progression of benign prostatic hyperplasia in asymptomatic men with enlarged prostate: a post hoc analysis of the REDUCE study. BMJ 346: f2109.

10. Liptay S, Bachem M, Häcker G, Adler G, Debatin KM, et al. (1999] Inhibition of nuclear factor kappa B and induction of apoptosis in T-lymphocytes by sulfasalazine. Br J Pharmacol 128(7): 1361-1369.

11. Baiker (2014) United States Patent Application Publication (10) Pub. No.: US 2004/0198826A1 (43) Pub.

12. Perletti G, Skerk V, Magri V, Markotic A, Mazzoli S, et al. (2011) Macrolides for the treatment of chronic bacterial prostatitis: an effective application of their unique pharmacokinetic and pharmacodynamic profile (Review). Mol Med Rep 4(6): 1035-1044.

13. Ikeuchi T, (1990) Clinical studies on chronic prostatitis and prostatitislike syndrome. (3). Clinical re-examination of chronic bacterial prostatitis using the criteria for clinical evaluation].Clinical studies on chronic prostatitis and prostatitis-like syndrome. Evaluation of prostatitis complicated by anal disease. Hinyokika Kiyo 36(5): 561568.

14. Takechi S, Yokoyama M, Tanji N, Nishio S, Araki N (1999) Nonbacterial prostatitis caused by partial urethral obstruction in the rat. Urol Res 27(5): 346-350

15. Choi H, Chang YS, Park BH, Ko DH, Moon YJ, et al. (2012) Comparison of Clinical Efficacy of Finasteride andDutasteride as 5-alpha Reductase Inhibitor Korean J 30(1).

16. (2013) J Clin Pharm Ther. 38(5): 405-415. 
Your next submission with Juniper Publishers will reach you the below assets

- Quality Editorial service

- Swift Peer Review

- Reprints availability

- E-prints Service

- Manuscript Podcast for convenient understanding

- Global attainment for your research

- Manuscript accessibility in different formats

( Pdf, E-pub, Full Text, Audio)

- Unceasing customer service

Track the below URL for one-step submission https://juniperpublishers.com/online-submission.php 